\title{
SENN, ON THE SURGERY OF THE PANCREAS.1
}

In an elaborate memoir presented at the 1886 meeting of the American Surgical Association, the author, Prof. N. Senn, has attempted to supply some data upon which to base rational methods of treatment for such of the injuries and diseases of the pancreas as may possibly be amenable to surgical interference. His work has included clinical researches and practical experiments, in the latter of which, dogs and cats were used exclusively. In all the experiments the most careful antiseptic precautions were carried out; the greater omentum was either pushed forward or, in the majority of cases, an opening was made into it by tearing at a point opposite the intestinal incision. The guide to the pancreas was always the pyloric orifice of the stomach. After the index finger had reached this point, it was passed along the duodenum for three or four inches, when the bowel was grasped between the index finger and thumb and brought into the incision with the pancreas.

Wounds of the Pancreas.-As the result of several experiments upon complete section of the gland, the author concludes that even when the ends are kept in accurate coaptation, complete section of the duct appears to result uniformly in the obliteration of the duct at the site of section. The obliteration is the direct result of the formation of a cicatrix in the lumen of the duct from the cut surfaces. The practical deductions are that in transverse risceral wounds of the pancreas, the most important indication that presents itself is to arrest hemorrhage by ligaturing the bleeding vessels, and to resort to suturing of the severed organ with a view to retain both ends of the pancreas as nearly as possible in their normal location, and thus maintain as nearly as possible the integrity of the vascular supply, rather than with a purpose to obtain restoration of continuity of the divided pancreatic duct, which, if it could be accomplished, would preserve the physiological importance of the detached portion of the gland. By detached portion of the gland is meant that portion which no longer remains in

'The Surgery of the Pancreas, as based upon experiments and clinical researches. By Nicholas Senn, M. D., of Milwaukee, Wis. Transactions of the American Surgical Association, 1886 : also International Josurnal of the Nedical Sciences 
physiological connection with the intestine and which never regains its physiological impoitance after the duct has become obliterated by a cicatrix at the point of section.

In an experiment upon the result of laceration of the pancreas, the organ being torn completely across and dropped into the abdominal cavity, hæmorrhage was arrested spontaneously, and the process of repair, so far as the wound in the pancreas was concerned, appeared to be satisfactory, but death resulted from the accidental opening of the wound with prolapse, strangulation and gangrene of the duodenum.

It having been asserted by a number of authorities that dead pancreatic tissue is a highly putrescible substance, and on this account its presence is very liable to serve as a source of infection, the pancreas was crushed in two cats to test the correctness of this observation; one being in poor condition, died; but the other lived in good health until it was killed. In both instances the crushed parenchyma of the organ was promptly removed by absorption, which seems in this particular locality to proceed with unusual activity, an occurrence which can only be explained by the assumption that the peritoneum is active in the process. No infection took place and no evidences of putrefaction could be found. Should wound infection take place in cases of this kind, there can be no doubt that the dead pancreatic tissue would serve as a most favorable soil for the septic germs, and would thus create the most essential condition for a rapid and most dangerous form of infection. Subcutaneous crushing or comminution of the pancreas then is in itself not a fatal or even dangerous injury.

Extirpation of the Pancreas, Complite or Partial.-In six cases the entire pancreas was extirpated with a fatal result in every instance in from a few hours to nine days after the operation, the cause of death being either the primary effects of the traumatism, hæmorrhage and shock, or secondary pathological lesions traceable directly to the operatıon, peritonitis and gangrene of the duodenum. Contrary to the opinion of Schiff, in dogs and cats at least, this operation is necessarily fatal.

Partial extirpation of the pancreas implies a less degree of trauma- 
tism, and consequently less danger of causing serious nutritive changes in adjacent organs than complete extirpation, and for these reasons it is less dangerous in a strictly surgical sense, although physiologically it may imply the same consequence as complete extirpation. as when the portion of the gland removed embraces the common duct or both principal ducts from each portion of the gland. Of four experimental operations, in two only the dogs lived for a sufficient length of time to determine the influence of the pancreatic secretion upon digestion and assimilation, the general health and nutrition remaining unimpaired for four weeks, when emaciation with fatty stools followed, which resulted in death from marasmus in one after seventy.six days, and reduced the second to a skeleton in one hundred and twenty-six days. These experiments would then tend to prove that the pancreatic secretion is an important, if not essential digestive fluid, and that in cases where no pancreatic juice can enter the intestine, or where secretion is entirely suspended, digestion and assimilation become impaired in all cases where the supposed vicarious action of other organs is inadequate to perform the functions of the extirpated or degenerated pancreas.

Fourteen experinents demonstrate the feasibility of ligature of either poition of the pancreas near the common duct as a surgical procedure. and the regularity with which the pancreatic tissue is removed by degeneration and absorption in the detached portion of the gland. After ligature of the duct or gland, secretion continues, and as the space for accumulation of fluid is limited, a certain degree of pressure within the duct is established, as is evident from the uniformity with which the ducts throughout that portion of the gland were found dilated. In no instance, however, was anything observed which resembled a cyst. The dilatation was not limited to any particular portion of the duct; it always presented itself as a unifonn ectasia of the entire duct. We can only explain the moderate dilatation by assuming that, as soon as a certain degree of pressure is reached, the pancreatic juice is removed by absorption by the vessels and lymphatics of the pancreas, and that a greater accumulation of fluid and distension of the duct could only occur when this function has become diminished or suspended by or- 
ganic changes in the structures which are concerned in the removal of the secretions. The atrophic changes in the parenchyma of the detached portion of the gland cannot be satisfactorily explained. Practically, this observation is of great importance. because it demonstrates that in operations upon the pancreas it is not essential or necessary to remove peripheral portions of the gland for fear that if any of the parenchymatous structures should remain, a retention cyst would follow. In partial resections for injury or disease, it would be advisable to ligature the peripheral portion, and permit it to remain, 25 it would lessen the danger by the infliction of less traumatism, and we can confidently expect that it will be removed in a short time by absorption. These experiments also settle definitely an important pathological question. It has been claimed by all writers that cysts of the pancreas are produced by obstruction of the common duct. In most of the specimens which have been examined, it is distinctly stated that obstruction was not complete, as for instance in cases of impaction of pancreatic calculi, when found in connection with cysts. In all of these experiments, obstruction of the duct was sudden and complete by elastic constriction, and subsequently permanent by the formation of a cicatrix between the divided ends of the duct. In none of the specimens, where life was sufficiently prolonged, did the process of obliteration fail to take place, and yet in none of them was even an attempt at the formation of a cyst observed. Experiments with the double ligature teach the importance of removing such portions of the pancreas as are not supplied with blood vessels, rather than trust to the doubtful expedient of leaving them to be removed by absorption, as dead pancreatic tissue is an exceedingly putrescible substance and furnishes the most favorable conditions for the growth and increase of septic germs.

Experiments made for the distinct purpose of studying the functional activity of a detached portion of the pancreas demonstrated conclusively that when a portion of the pancreas is detached by complete section, secretion continues until, by degeneration and ab. sorption, the parenchyma of the gland has disappeared. That the atrophy in the part of the organ which had been detached from its connections with the intestines was not due to a traumatic interstitial 
pancreatitis, is proved by the normal appearance and structure of the remaining portion of the gland which had retained its anatomical and physiological relations to the intestine, supporting the assertion that physiological detachment of any portion of the pancreas is invariably followed by degeneration and complete atrophy, consequently also by complete cessation of functional activity.

It is a well known fact that the pancreatic juice has an irritating action upon the skin, and eleven experiments were undertaken to discover its action upon the peritoneum, by the formation of internal pancreatic fistulx. As the secretions often amount to more than four ounces a day in external pancreatic fistula, it is reasonable to believe that the same quantity was discharged into the peritoneal cavity and that in the nine healthy cases, two dying with purulent peritonitis, it was absorbed by the oeritoneum. These, as well as the ligature experiments, corroborate the statement made by some authors that the introduction of normal pancreatic juice into the circulation is innocu. ous and that this abnormal supply is tolerated for two weeks or more without any appreciable ill consequences."

Of all abdominal organs, the pancreas is most exempt from injurg; both from indirect and direct violence, a circumstance entirely due to its remote location and the ample protection furnished by the rertebral column and the bony walls of the chest. The anatomical relations of the pancreas with numerous and important organs are such that when this organ is injured, the same violence is apt to have wounded an adjacent and perhaps more important viscus. And the trequency of such grave complications with the profuse hremorrhage usually attending such injury impart to wounds of the pancreas more than ordinary gravity. The author has collected thirteen cases of wound of the pancreas, eight of which were fatal. In not a single case did symptoms point first to this organ as the seat of lesion. If such a lesion is found upon exploratory laparotomy, it should certainly receive treatment. In case of extensive crushing, it would be good surgery to remove the crushed portion after preliminary ligature of the organ on each side of the comminuted portion. Ligature of the pancreas can be safely done with a single catgut or silk ligature as the fri- 
able texture of the organ will permit of burying the ligature deeply, a circumstance which will guard against its slipping. If the pancreas is lacerated, each end of the organ should be ligatured for the purpose of arresting or preventing hremorrhage, as well as to guard against extravasation of pancreatic juice into the abdominal cavity. Notwithstanding the favorable results of the exudation of pancreatic juice in the abdominal cavity in his experiments upon animals, the author considers that there can be no doubt that the presence of crushed pancreatic tissue and pancreatic juice in the peritoneal cavity after abdominal section, would greatly enhance the danger of traumatic infection, and for this reason, if for no other, the former should be removed and the escape of the latter prevented by ligature of the pancreas on the side or each side of the crushed or lacerated portion.

Prolapse of the Pancreas.-The treatment of prolapse of the pancreas will depend upon the pathological condition of the viscus at the time the patient comes under the care of the surgeon. If the prolapse is recent and the organ presents no indication of inflammatory or other changes, it should be thoroughly disinfected and replaced. It is of the greatest importance not to resort to violence in effecting reduction, as irreparable damage may be inflicted by resorting to more than the gentlest force. When reduction is not readily accomplished, the wound should be enlarged. If the pancreas is in a condition of inflammation or gangrene, the parts should be thoroughly disinfected and the organ pulled further into the wound until healthy tissue is reached, when a ligature is applied and the diseased portion removed with the knife or scissors. After another thorough disinfection, the stump is dropped into the abdominal cavity and the external wound closed. Thorough primary removal of infected tissue is the only safety against subsequent extension of the infection to the peritoneal carity, and the only guarantee for primary union of the abdominal wound.

Gunshot Injuries of the Pancreas, when they come under the observation of the surgeon as an independent lesion or as a complication of other visceral injuries in case of penetrating wounds of the abdomen treated by laparotomy, should be treated in the same way as a contusion or laceration of the gland. 
While the surgeon may unhesitatingly remove the tail and a portion of the body of the pancreas without fear of any immediate or remote ill effects, great care must be exercised in operating in the vicinity of the head, to preserve the integrity of the common duct and as much of this portion of the organ as may appear compatible with the condition which necessitates the operation.

Inflammation of the Pancreas.-Referring briefly to acute pancreatitis, he passes on to chronic interstitial pancreatitis or sclerosis of the gland, which sometimes produces stenosis of the bile-duct or the pancreatic duct and, when obstruction is followed by retention of the secretions, an operation always becomes necessary in biliary retention, which should be treated by establishing a new outlet for the bile into the duodenum, while the formation of an external pancreatic fistula in cases of cyst of the pancreas becomes necessary only when the presence of the swelling in' itself has become a source of sufficient pain and discomfort to warrant treatment by abdominal section.

One of the terminations of acute inflammation of the pancreas is gangrene. Cases have been reported where spontaneous recovery followed elimination of the necrosed organ through the alimentary canal. If spontaneous recovery in this condition is possible, it would seem plausible that a timely removal of the necrosed organ by surgical in. terference would add to the chances of recovery. Consequently gangrene is added to the diseases of the pancreas which should be treated by operative measures. The pancreas may constitute one of the component parts of the intussusception in cases of invagination of the borrels. In searching for the cause of intestinal obstruction and peritonitis, during laparotomy, the pancreas should not be forgotten, and when it is found that the primary disease is located in or around this organ, radical mensures should be adopted whenever such a course appears practicable. Whenever the sac can be stitchcd to the external incision, this should be done and the sac opened, disinfected and drained. Search should be made for the necrosed pancreas, and when found detached, it should be removed. As in most of these cases, the retroperitoncal tissue is excessively infiltrated, a counter-opening should be made in the lumbar region and through drainage estab- 
lished. If an anterior abdominal fistula cannot be established, the course to be pursued should be the same as in treating a pancreatic abscess under similar conditions.

Absecss of Pancreas.-There can be no doubt that in the near future abscesss of the pancreas will be treated on the same principles as suppuration in any other locality. The remote location of the abscess may offer many serious obstacles to diagnosis and a rational course of treatment, but these difficulties will be overcome by improved methods of examination and more perfect methods of operation. As suppuration is only one of the terminations of inflammation, abscess, like in. flammation, may occur primarily in the gland itself, or it may com. mence in the para- or peri-pancreatic tissue. If the abscess is endo-pancreatic, it may be bounded and circumscribed by the proper investment of the gland. If, on the other hand, it commences primarily outside of the gland, it appears as a diffuse abscess, which extends to the pancreas by contiguity; in other words, we speak of the abscess as a suppurative pancreatitis or a suppurative peri- or parapancreatitis.

As primary, idiopathic, uncomplicated purulent inflammation of the pancreas is an exceedingly rare affection, it is of great practical importance in the surgical treatment of such cases to determine, if pos. sible, the predisposing cause or causes and to ren.ove them or render them inert at the time of operation.

The presence of pus within the pancreas or its immediate vicinity is not indicated by any characteristic or positive symptoms. The symptoms always point to the stomach or liver as the seat of the disease. The most prominent and constant symptoms which have been observed are nausea, vomiting of a clear, greenish or viscid fluid, thirst, loss of appetite, constipation, progressive emaciation and distension of the epigastrium. If the abscess is large, it will be recognized by palpation and deep percussion as a tumor in the epigastric region. In such cases a probable diagnosis may always be made by careful and systematic physical examination and reasoning by exclution. Finally, in all cases where a tumor can be felt in the epigastric region and a probable diagnosis can be made regarding its benign character, an 
exploratory laparotomy should be resorted to for the purpose of making an accurate anatomical diagnosis. The prognosis of abscess of the pancreas is always unfavorable. Death is produced by progressive emaciation and inanition, by septic absorption or secondary lesions in adjacent organs. The treatment indicated is incision, evacuation and antiseptic irrigation and drainage, where needed.

Hamorrlage of the Pancreas.-A number of cases of hemorrhage of the pancreas are presented and discussed. The author considers that the propriety of surgical treatment of pathological hxmorrhage of the pancreas can only be entertained when the accident takes place in consequence of circumscribed benign pathological conditions which in themselves do not jeopardize the life of the patient, and which admit of measures for arrcsting hremorrhage by direct treatment. Operative interference should, therefore, be limited to hremorrhagic cysts of the pancreas, in well-defined cases of which it would be justifiable to resort to abdominal section as the only means of arresting fatal hæmorrhage, by direct ligaturc of the bleeding points, or by removing the localized portions of diseased tissue from which the hxmorrhage has taken place. The same treatment is applicable to diffuse hiemorrhag e due to a localized lesion.

Cysts of the Pancreas.-The remarks on cyst of the pancreas are merely supplementary to the author's previous paper on the subject (vid. ANNals of Surgery. Vol. ii, p. 272). The most important etiological factor in these cases must be sought for in an arrest of absorption of the pancreatic juice due either to a transformation of that fluid by the admixture of pathological products into a substance which is incapable of being absorbed, or to a loss of function in this direction of the vcssels which perform this task. Extirpation of the cyst would guard most effectually against the formation of a permanent pancreatic fistula, but on account of the deep location of the pancreas, shortness or absence of a pedicle, and the many obstacles thrown in the way of the operator by adjacent organs, the procedure becomes one surrounded by innumerable difficulties and, in the present state of our science, of doubtful propriety. The formation of an external pan. creatic fistula in the treatment of cysts of the pancreas has been so 
uniformly successful that it should be iovariably adopted io preference to excision, aod the latter operatioo should only be resorted to io cases where portions of the cyst wall have become the seat of malignaot disease; likewise io cases where life is threatened by hremorrhage into a cyst by rupture of the vessels lining its interior and which cannot be controlled by simpler and less hazardous measures.

Neoplasms.-Referring briefly to hypertrophy of the pancreas and quoting one doubtful case, he passes on to cancer of the pancreas, ooting the uncertainty of the symptoms in the diagnosis of this affection. A satisfactory conclusion can be reached only after a careful coosideration of the history of the case combined with a systematic elucidation of all the symptoms presentcd, and more particularly by resorting to the advantages to be derived from a systematic and careful study by exclusion. A positive diagnosis of malignant disease of the pancreas is only possible after the tumor has attained sufficient size to be recognizable by palpation, and consequently too late for a radical extirpation. When the disease has advanced to this stage, it has already involved the greater portion of the gland and, as a rule, has invaded important adjacent organs. Another important element in the surgical treatment of cancer of the pancreas consists in the fact that the disease, as a rule, develops primarily in the head of the organ, a location which, in itself, precludes the propriety of an operation. The most favorable conditioos for extipation are presented if the disease is primarily located in the tail of the pancreas and has not passed beyood the limits of the capsule of the gland. In such a case, excision of the splenic extremity would offer a fair prospect for a permaoeot result without eodangering, as a remote coosequence, the process of digestion, as a sufficient amount of secretiog structure would remain in conoection with the intestine to maintain pancreatic digestion.

Passing lightly over tuberculosis of the pancreas and lipomatosis of the gland, both of which are extremely rare affections, he dwells rither more at length upon lithiasis of the pancreatic ducts, a frequent affectioo, the positive diagoosis of which duriog life is impossible, and the surgical treatmeot of which is consequeotly limited to the manage- 
ment of some of its consecutive lesions-cysts, abscess and retention of bile, and concludes with the following propositions:

(1). Restoration of the continuity of the pancreatic duct did not take place after complete section of the pancreas. (2). Complete extirpation of the pancreas was invariably followcd by death, produced . either by the traumatism or gangrene of the duodenum. (3). Partial excision of the pancreas for injury or disease was a feasible and justifiable surgical procedure. (4). Complete obstruction of the pancreatic duct, uncomplicated by pathological conditions of the parenchyma of the organ, never resulted in the formation of a cyst. (5). In simple cbstruction of the pancreatic duct the pancreatic juice was removed by absorption. (6). Gradual atrophy of the pancreas from nutritive or degenerative changes of the secreting structure was not incompatible with health. (7). Physiological detachment of any portion of the pancreas was invariably followed by progressive degeneration of the glandular tissue. (8). Extravasation of pancreatic juice into the peritoneal cavity did not produce peritonitis. (S). Crushed or lacerated pancreatic tissue was removed by absorption, provided the site of operation remained aseptic. (10). Complete division of the pancreas by elastic constriction was never followed by restoration of interrupted anatomical continuities. (II). Limited detachment of the mesentery from the duodenum, as required in operations upon the pancreas, was not followed by gangrene of the bowel. (12). In all operations upon the head of the pancreas the physiological attachment of the peripheral portion of the gland should be maintained by preserving the ir.tegrity of the main pancreatic duct. (13). Partial excision of the splenic portion of the pancreas was indicated in cases of circumscribed abscess and malignant tumors in all cases where the pathological product could be removed completely without danger of compromising pancreatic digestion, or of inflicting additional injury upon important adjacent organs. (14). Ligation of the pancreas at the point or points of section should precede extirpation as a prophylactic measure against troublesome hæmorrhage and the extravasation of pancreatic juice into the peritoneal canty. (15). The formation of an external pancreatic fistula by abdominal section was indicated in 
the treatment of cysts, abscess, gangrene and hæmorrhage of the pancreas due to local causes. (16). Abdominal section and lumbar drainage were indicated in cases of abscess or gangrene of the pancreas where it was found impossible to establish an anterior abdominal fistula. (17). Through-drainage was indicated in cases of abscess and gangrene of the pancreas, with diffuse burrowing of pus in the retroperitoneal space. (18). Removal of an impacted pancreatic calculus in the duodenal extremity of the duct of Wirsung by taxis, or incision and extraction, should be practised in all cases where the common bileduct was compressed or obstructed by the calculus, and death was threatened by cholremia. (19). In such cases the principal source of danger, extravasation of bile into the peritoneal cavity, should be avoided by preliminary aspiration of the dilated bile-ducts, accurate closure of the visceral wound with fine silk sutures, and absolute physiological rest of the organs of digestion during the time required for the healing of the visceral wound.

JaMes E. Pilcher. 